\title{
PS-004 REVIEW OF HIV IN SUB-SAHARAN AFRICA: CURRENT SITUATION, OPPORTUNITIES AND CHALLENGES
}

Catherine Hankins. Amsterdam Institute for Global Health and Development, The Netherlands

\subsection{6/bmjgh-2016-000260.4}

UNAIDS estimates that in 2015, 19 million (17.7-20.5 million) and 6.5 million (5.3-7.8 million) people were living with HIV in Eastern and Southern Africa (ESA) and Western and Central Africa (WCA), respectively. Between 2010 and 2015, new HIV infections declined by $14 \%$ in ESA and $8 \%$ in WCA, while AIDS-related deaths fell by $38 \%$ and $10 \%$, respectively. In ESA, 10.3 million people or $54 \%(50-58 \%)$ of all people living with HIV were accessing antiretroviral therapy (ART), compared to 1.8 million people or 28\% (23-34\%) of all people living with HIV in WCA. Since 2010, there has been a $66 \%$ decline in new $\mathrm{HIV}$ infections among children in ESA compared to a $31 \%$ decline in WCA.

These striking regional differences mask large discrepancies in country progress, sex differences in ART uptake, and a diversity of micro-epidemics across sub-Saharan Africa. The goal to end AIDS as a public health threat by 2030, the UN-AIDS 90-90-90 treatment cascade goal for 2020, and the WHO recommendation to offer ART when HIV is diagnosed regardless of CD4 count are galvanizing public health and community-based responses in Africa. Despite improvements in ART and new prevention tools, including voluntary medical male circumcision and oral preexposure prophylaxis, the goals will not be achieved without new tools, including an HIV vaccine and a cure.

EDCTP has funded high-impact HIV research that has resulted in, among others, policy change; prequalification of new products; improved treatment strategies, including for children; and strategies to prevent mother-to-child transmission, while building research capacity and clinical trial infrastructures. Promoting African country membership and increased financial contributions from African countries, EDCTP2 aims to leverage co-funding from public/private sources for calls for proposals that address important gaps in HIV prevention and treatment science that can be answered through phase I-IV clinical trials in sub-Saharan Africa. 